\title{
Sparse Array Angle Estimation Using Reduced-Dimension ESPRIT-MUSIC in MIMO Radar
}

\author{
Chaozhu Zhang and Yucai Pang \\ College of Information and Communication Engineering, Harbin Engineering University, Harbin 150001, China \\ Correspondence should be addressed to Yucai Pang; pangyucai@yeah.net
}

Received 17 September 2013; Accepted 14 October 2013

Academic Editors: E. A. Marengo, K. Teh, and A. Torsello

Copyright (c) 2013 C. Zhang and Y. Pang. This is an open access article distributed under the Creative Commons Attribution License, which permits unrestricted use, distribution, and reproduction in any medium, provided the original work is properly cited.

\begin{abstract}
Sparse linear arrays provide better performance than the filled linear arrays in terms of angle estimation and resolution with reduced size and low cost. However, they are subject to manifold ambiguity. In this paper, both the transmit array and receive array are sparse linear arrays in the bistatic MIMO radar. Firstly, we present an ESPRIT-MUSIC method in which ESPRIT algorithm is used to obtain ambiguous angle estimates. The disambiguation algorithm uses MUSIC-based procedure to identify the true direction cosine estimate from a set of ambiguous candidate estimates. The paired transmit angle and receive angle can be estimated and the manifold ambiguity can be solved. However, the proposed algorithm has high computational complexity due to the requirement of two-dimension search. Further, the Reduced-Dimension ESPRIT-MUSIC (RD-ESPRIT-MUSIC) is proposed to reduce the complexity of the algorithm. And the RD-ESPRIT-MUSIC only demands one-dimension search. Simulation results demonstrate the effectiveness of the method.
\end{abstract}

\section{Introduction}

MIMO radar employs multiple transmit and receive elements and has the ability to jointly plan transmissions and process received signals. It has recently been the focus of research owing to its significant performance improvement compared to the conventional phased-array radar [1-3]. MIMO radar has more degrees of freedom than other systems with a single transmit element. These additional degrees of freedom are well-qualified for overcoming fading effect, enhancing spatial resolution, strengthening parameter identifiability, and also improving target detection performance [4-6].

In order to estimate the transmit angle and receive angle in the bistatic MIMO radar, some methods have been proposed $[7,8]$. However, both of them cannot be applied in sparse linear arrays. In [9], the authors discuss the imaging method of the moving targets in MIMO radar with sparse array. Also, the issue of sparsity in the specific context of a MIMO radar system is studied in [10]. Sparse linear arrays can provide a large measurement basis with a reduced number of elements as compared to filled arrays. But the disadvantage of sparse array is manifold ambiguity which can cause large angle estimation errors. In [11, 12] the authors discuss the angle estimation performance for different sparse linear array configurations using the Ziv-Zakai bound (ZZB). They illustrate that the ZZB includes three terms which correspond to the three types of estimation errors: random errors, small main lobe errors, and errors due to side lobe ambiguities.

In conventional radar, three types of methods are proposed to solve the ambiguity problem in angle estimation. The first type is to change the original array by inducing an additional sensor or sliding the sensors positions [13-15]. The second type is to optimize the sensor positions of an array [16-18]. The last type focuses on the algorithms rather than on the hardware [19-21].

In this paper, we present an angle estimation method based on ESPRIT and MUSIC to avoid the manifold ambiguity for the bistatic MIMO radar spaced sparse linear arrays. The main idea of the method uses MUSIC-based procedure to search the true direction cosine estimate from a set of ambiguous candidate estimates. And the ambiguous candidate estimates are obtained by ESPRIT algorithm. But, the requirement of two-dimension search renders much higher computational cost. Then we derive a Reduced-Dimension ESPRIT-MUSIC algorithm which reduces the complexity for angle estimation in the bistatic MIMO radar with sparse 


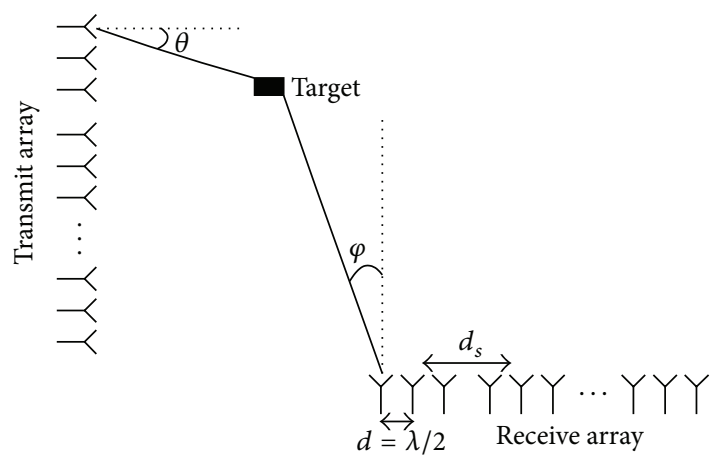

FIGURE 1: Bistatic MIMO radar with sparse linear arrays.

linear arrays. Compared to the uniform linear array (ULA), we can obtain more accurate angle estimation.

\section{Problem Formulation}

As shown in Figure 1, a bistatic MIMO radar system consists of $M_{t}$-element transmit array and $M_{r}$-element receive array, both of which are spaced sparse linear arrays. The transmit array is composed of $N_{s t}$ subarrays with $M_{s t}$ sensors per subarray, and the receive array is composed of $N_{s r}$ subarrays with $M_{s r}$ sensors per subarray, intersensor spacing $d=\lambda / 2$ and intersubarray spacing $d_{s} \gg d$. The targets are assumed to be in the far-field of transmit and receive arrays. At the transmit site, $M_{t}$ different narrowband signals are emitted simultaneously, which have identical bandwidth and centre frequency but are temporally orthogonal. Assume that $P$ noncoherent targets, respectively, correspond to the transmit angle $\theta_{p}$ and the receive angle $\varphi_{p}$, where $p=1, \ldots, P$, are presented in the same range. For convenience, we consider that estimation of angle cosines are relative to the array axis $\mu_{p}=\sin \theta_{p}, \nu_{p}=\sin \varphi_{p}$.

The output of the entire matched filters at the receiver can be expressed as

$$
\mathbf{x}(t)=\mathbf{A s}(t)+\mathbf{n}(t),
$$

where $\mathbf{A}=\left[\mathbf{a}_{1}, \mathbf{a}_{2}, \ldots, \mathbf{a}_{P}\right]$ is an $M_{t} M_{r} \times P$ matrix composed of the $P$ steering vectors and $\mathbf{a}_{p}=\mathbf{a}\left(v_{p}\right) \otimes \mathbf{a}\left(\mu_{p}\right)$ is the Kronecker product of the receive and the transmit steering vectors for the $p$ th target.

And the transmit steering vectors can be written as

$$
\mathbf{a}\left(\mu_{p}\right)=\mathbf{a}_{s}\left(\mu_{p}\right) \otimes \mathbf{a}_{l}\left(\mu_{p}\right),
$$

where $\mathbf{a}_{l}\left(\mu_{p}\right)$ is the steering vector of the transmit subarray which is defined by

$$
\mathbf{a}_{l}\left(\mu_{p}\right)=\left[1, e^{j \omega_{t p}}, \ldots, e^{j\left(M_{s t}-1\right) \omega_{t p}}\right]^{T},
$$

where $\omega_{t p}=(2 \pi / \lambda) d \mu_{p},(\cdot)^{T}$ stands for transpose and $\mathbf{a}_{s}\left(\mu_{p}\right)$ is the vector describing the phase shifts caused by transmit subarray displacements

$$
\mathbf{a}_{s}\left(\mu_{p}\right)=\left[1, e^{j \omega_{t p}^{a}}, \ldots, e^{j\left(N_{s t}-1\right) \omega_{t p}^{a}}\right]^{T},
$$

where $\omega_{t p}^{a}=(2 \pi / \lambda) d_{s} \mu_{p}$.
Also, the receive steering vectors can be expressed as

$$
\mathbf{a}\left(v_{p}\right)=\mathbf{a}_{s}\left(v_{p}\right) \otimes \mathbf{a}_{l}\left(v_{p}\right),
$$

where $\mathbf{a}_{l}\left(v_{p}\right)$ is the steering vector of the receive subarray which is defined by

$$
\mathbf{a}_{l}\left(v_{p}\right)=\left[1, e^{j \omega_{r p}}, \ldots, e^{j\left(M_{s r}-1\right) \omega_{r p}}\right]^{T},
$$

where $\omega_{r p}=(2 \pi / \lambda) d v_{p}$ and $\mathbf{a}_{s}\left(\nu_{p}\right)$ is the vector describing the phase shifts caused by receive subarray displacements

$$
\mathbf{a}_{s}\left(v_{p}\right)=\left[1, e^{j \omega_{r p}^{a}}, \ldots, e^{j\left(N_{s r}-1\right) \omega_{r p}^{a}}\right]^{T},
$$

where $\omega_{r p}^{a}=(2 \pi / \lambda) d_{s} v_{p}$.

$\mathbf{s}(t)=\left[s_{1}(t), s_{2}(t), \ldots, s_{P}(t)\right]^{T}$ is a column vector consisting of the phase and amplitudes of the $P$ sources at time $t$ which is usually in the form of $s_{p}=\alpha_{p} e^{j \omega_{d p} t}$ with $\omega_{d p}$ being the Doppler frequency and $\alpha_{p}$ the amplitude involving the reflection coefficients and path losses and so on. The $M_{t} M_{r} \times 1$ noise vector $\mathbf{n}(t)$ is assumed to be independent, zero-mean complex Gaussian distribution.

\section{ESPRIT-MUSIC for Angle Estimation}

3.1. The Theory of ESPRIT-MUSIC Algorithm. The maximum likelihood (ML) estimation of the covariance of $\mathbf{x}(t)$ for $L$ snapshots is $\mathbf{R}=1 / L \sum_{l=1}^{L} \mathbf{x}\left(t_{l}\right) \mathbf{x}^{H}\left(t_{l}\right)$, where $[\cdot]^{H}$ denotes the Hermitian transpose. Let $\mathbf{E}_{s}$ be the $M_{t} M_{r} \times P$ signal subspace matrix composed of the $P$ eigenvectors corresponding to the largest $P$ eigenvalues of $\mathbf{R}$, and the last $M_{t} M_{r}-P$ eigenvectors constitute the noise subspace $\mathbf{E}_{n}$. It can be shown that $\mathbf{A}$ and $\mathbf{E}_{s}$ span the same subspace. Therefore, there exists a unique nonsingular $\mathbf{T}$ such that $\mathbf{E}_{s}=\mathbf{A T}$. We define a new $M_{t} M_{r} \times P$ matrix $\mathbf{A}^{\prime}=\left[\mathbf{a}_{1}^{\prime}, \mathbf{a}_{2}^{\prime}, \ldots, \mathbf{a}_{P}^{\prime}\right]$, where $\mathbf{a}_{P}^{\prime}=\mathbf{a}\left(\mu_{p}\right) \otimes \mathbf{a}\left(\nu_{p}\right)$. Then the matrix $\mathbf{A}^{\prime}$ is row equivalent to $\mathbf{A}$. $\mathbf{E}_{s}^{\prime}$ is an $M_{t} M_{r} \times P$ signal subspace matrix formed from $\mathbf{E}_{s}$ by the same row interchange operations as $\mathbf{A}^{\prime}$ is formed from $\mathbf{A}$. Let $\mathbf{A}_{r 1}$ and $\mathbf{A}_{r 2}$ be the $M_{s t} N_{s t} M_{s r}\left(N_{s r}-1\right) \times P$ submatrices of $\mathbf{A}$ consisting of the first and the last $M_{s t} N_{s t} M_{s r}\left(N_{s r}-1\right)$ rows of $\mathbf{A}$, respectively. Then $\mathbf{A}_{r 2}=\mathbf{A}_{r 1} \boldsymbol{\Phi}_{r}$, where $\boldsymbol{\Phi}_{r}=\operatorname{diag}\left[e^{j \omega_{r 1}^{a}}, e^{j \omega_{r 2}^{a}}, \ldots, e^{j \omega_{r P}^{a}}\right]$. Here $\operatorname{diag}(\mathbf{b})$ denotes a diagonal matrix constructed by the vector $\mathbf{b}$. Let $\mathbf{E}_{r 1}$ and $\mathbf{E}_{r 2}$ be the $M_{s t} N_{s t} M_{s r}\left(N_{s r}-1\right) \times P$ submatrices formed from $\mathbf{E}_{s}$ in the same way as the $\mathbf{A}_{r 1}$ and $\mathbf{A}_{r 2}$ are formed from $\mathbf{A}$. Then the diagonal elements of $\boldsymbol{\Phi}_{r}$ are the eigenvalues of $\boldsymbol{\Psi}_{r}=\mathbf{T}^{-1} \boldsymbol{\Phi}_{r} \mathbf{T}$, which satisfy $\mathbf{E}_{r 2}=\mathbf{E}_{r 1} \boldsymbol{\Psi}_{r}$. The eigenvalue decomposition of $\boldsymbol{\Psi}_{r}$ yields $\widehat{\boldsymbol{\Phi}}_{r}=\mathbf{Q}^{-1} \boldsymbol{\Psi}_{r} \mathbf{Q}$, where $\widehat{\boldsymbol{\Phi}}_{r}$ is a diagonal matrix composed of the eigenvalues of $\boldsymbol{\Psi}_{r}$ and the columns of $\mathbf{Q}$ are eigenvectors of $\boldsymbol{\Psi}_{r}$. Thus, $\mathbf{A}^{\prime}=\mathbf{E}_{s}^{\prime} \mathbf{Q}$ [8]. Similarly, let $\mathbf{A}_{t 1}$ and $\mathbf{A}_{t 2}$ be the submatrices $M_{s t}\left(N_{s t}-1\right) M_{s r} N_{s r} \times P$ of $\mathbf{A}^{\prime}$ consisting of the first and the last $M_{s t}\left(N_{s t}-1\right) M_{s r} N_{s r}$ rows of $\mathbf{A}^{\prime}$. Then $\mathbf{A}_{t 2}=\mathbf{A}_{t 1} \boldsymbol{\Phi}_{t}$, where $\boldsymbol{\Phi}_{t}=\operatorname{diag}\left[e^{j \omega_{t 1}^{a}}, e^{j \omega_{t 2}^{a}}, \ldots, e^{j \omega_{t P}^{a}}\right]$. So we obtain $\hat{\xi}_{t p}^{a}=\exp \left(j \omega_{t p}^{a}\right)$, 
$\widehat{\xi}_{r p}^{a}=\exp \left(j \omega_{r p}^{a}\right)$, and the set of low-variance but cyclically ambiguous direction cosine estimates

$$
\begin{aligned}
& \widehat{\mu}_{p}^{a}=\frac{\arg \left(\hat{\xi}_{t p}^{a}\right)}{\left(2 \pi d_{s} / \lambda\right)}, \\
& \widehat{v}_{p}^{a}=\frac{\arg \left(\hat{\xi}_{r p}^{a}\right)}{\left(2 \pi d_{s} / \lambda\right)} .
\end{aligned}
$$

Because $d_{s} \gg d$, here exists a set of cyclically related candidates for the low-variance estimate of $\mu_{p}=\sin \theta_{p}, v_{p}=$ $\sin \varphi_{p}, p=1, \ldots, P$. It can be written as

$$
\begin{gathered}
\mu_{p}^{(l)}=\mu_{p}^{a}+l_{\mu}\left(\frac{\lambda}{d_{s}}\right), \\
v_{p}^{(l)}=v_{p}^{a}+l_{\nu}\left(\frac{\lambda}{d_{s}}\right) ;
\end{gathered}
$$

we define $l_{1}=\left(d_{s} / \lambda\right)\left(-1-\widehat{\mu}_{p}^{a}\right), l_{2}=\left(d_{s} / \lambda\right)\left(1-\widehat{\mu}_{p}^{a}\right), l_{3}=$ $\left(d_{s} / \lambda\right)\left(-1-\hat{v}_{p}^{a}\right)$, and $l_{4}=\left(d_{s} / \lambda\right)\left(1-\widehat{v}_{p}^{a}\right)$, the bounds for parameter $l_{\mu}, l_{\nu}$ are determined by

$$
\begin{aligned}
& \left\lceil l_{1}\right\rceil \leq l_{\mu} \leq\left\lfloor l_{2}\right\rfloor, \\
& \left\lceil l_{3}\right\rceil \leq l_{\nu} \leq\left\lfloor l_{4}\right\rfloor,
\end{aligned}
$$

where $\lceil z\rceil$ denotes the smallest integer greater than $z$ and $\lfloor z\rfloor$ denotes the largest integer less than $z$. The unambiguous estimates of direction cosines can be obtained based on the 2D-MUSIC null spectrum

$$
\begin{aligned}
& \left(\widehat{\mu}_{p}^{d}, \widehat{v}_{p}^{d}\right) \\
& =\underset{\mu_{p}^{(l)}, v_{p}^{(l)}}{\operatorname{argmin}}\left[\mathbf{a}_{p}^{H} \mathbf{E}_{n} \mathbf{E}_{n}^{H} \mathbf{a}_{p}\right] \\
& =\underset{\mu_{p}^{(l)}, v_{p}^{(l)}}{\operatorname{argmin}}\left[\left(\mathbf{a}\left(v_{p}\right) \otimes \mathbf{a}\left(\mu_{p}\right)\right)^{H} \mathbf{E}_{n} \mathbf{E}_{n}^{H}\left(\mathbf{a}\left(v_{p}\right) \otimes \mathbf{a}\left(\mu_{p}\right)\right)\right] .
\end{aligned}
$$

Then the paired transmit angle $\theta_{p}$ and receive angle $\varphi_{p}$ can be written as $\widehat{\theta}_{p}=a \sin \left(\widehat{\mu}_{p}^{d}\right) * 180 / \pi, \widehat{\varphi}_{p}=a \sin \left(\widehat{\nu}_{p}^{d}\right) * 180 / \pi$.

The major steps of ESPRIT-MUSIC algorithm for angle estimation in the bistatic MIMO radar with sparse linear arrays are as follows.

Step 1. Perform eigen-decomposition operation for the covariance matrix $\widehat{\mathbf{R}}$ to obtain $\mathbf{E}_{s}$ and $\mathbf{E}_{n}$.

Step 2. Calculate the ambiguous angle cosine estimates $\widehat{\mu}_{p}^{a}$ and $\widehat{v}_{p}^{a}$ via (8).

Step 3. Find the set of cyclically related candidates $\mu_{p}^{(l)}$ and $v_{p}^{(l)}$ by (9).

Step 4. Searching $\mu_{p} \in \mu_{p}^{(l)}, v_{p} \in v_{p}^{(l)}$ with respect to (11), the $P$ largest peaks $\left[\left(\widehat{\mu}_{1}^{d}, \widehat{\nu}_{1}^{d}\right),\left(\widehat{\mu}_{2}^{d}, \widehat{v}_{2}^{d}\right), \ldots,\left(\widehat{\mu}_{P}^{d}, \widehat{v}_{P}^{d}\right)\right]$ can be found. Then compute the transmit angle $\theta_{p}$ and the receive angle $\varphi_{p}$.

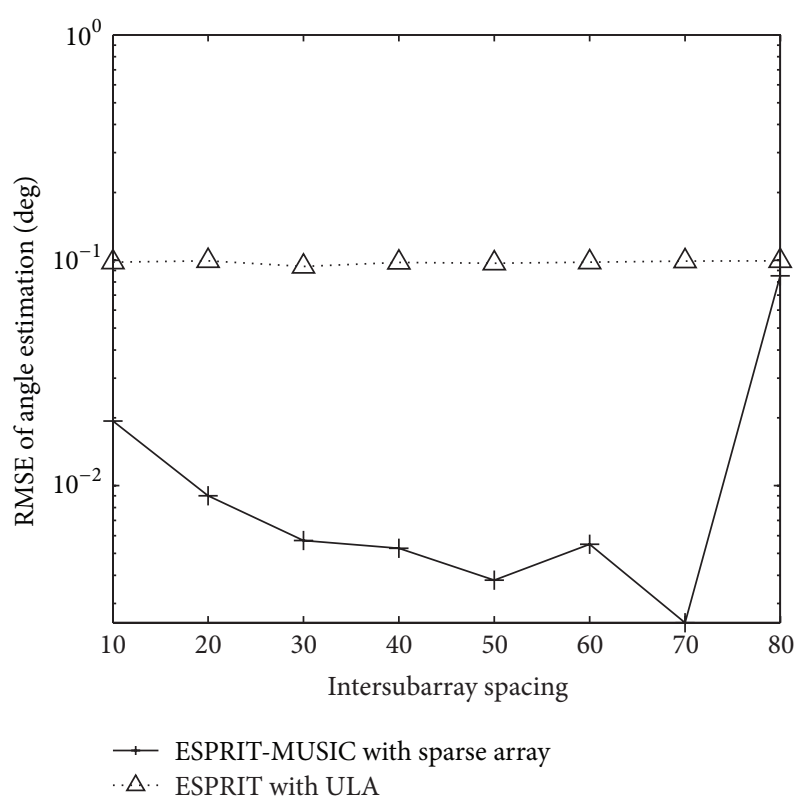

FIGURE 2: RMSE of angle estimation of the first target with different intersubarray spacing with $\mathrm{SNR}=4 \mathrm{~dB}$.

3.2. Simulation Results. We present 200 Monte Carlo simulations to demonstrate the angle estimation performance of ESPRIT-MUSIC algorithm. Assume that there exist $P=3$ uncorrelated stationary targets, which are located at angles $\left(\theta_{1}, \varphi_{1}\right)=\left(15^{\circ}, 20^{\circ}\right),\left(\theta_{2}, \varphi_{2}\right)=\left(25^{\circ}, 40^{\circ}\right)$, and $\left(\theta_{3}, \varphi_{3}\right)=$ $\left(10^{\circ}, 30^{\circ}\right)$, and the number of snapshots is $L$ for an $M_{t}=12$, $M_{s t}=3, N_{s t}=4$ and $M_{r}=10, M_{s r}=2, N_{s r}=5$ bistatic MIMO radar. The root mean squared error (RMSE) of the target angle estimation is defined as RMSE = $\sqrt{E\left(\hat{\theta}_{i}-\theta\right)^{2}+E\left(\widehat{\varphi}_{i}-\varphi\right)^{2}}$, where $\widehat{\theta}_{i}$ and $\widehat{\varphi}_{i}$ are the estimated transmit angle and the estimated receive angle of the same target for the ith-trial. Figure 2 shows the RMSE of angle estimation of the first target with different intersubarray spacing, $L=100, \mathrm{SNR}=4 \mathrm{~dB}$. The angle estimation performance significant degradation in 80 half-wavelengths can be seen. So, we select the intersubarray spacing $d_{s}=30 *(\lambda / 2)$ in the following simulation. Figure 3 presents the paired angle estimation results of ESPRIT-MUSIC algorithm for all three targets with $\mathrm{SNR}=0 \mathrm{~dB}, L=100$. The variation of angle estimation RMSE of the proposed algorithm and the method of [8] with SNR are shown in Figure 4. From Figures 3 and 4, we can see that the paired transmit angle and receive angle are correct, and the performances of ESPRIT-MUSIC with sparse array are much better than ESPRIT with ULA. Figure 5 depicts the algorithmic performance with different $L$. It illustrates that the angle estimation performance becomes better with $L$ increasing. From Figure 5, we also draw a conclusion that the proposed algorithm has a good performance with small sampling sizes, $L=30$.

\section{RD-ESPRIT-MUSIC for Angle Estimation}

4.1. The Theory of RD-ESPRIT-MUSIC Algorithm. ESPRITMUSIC algorithm requires two-dimension search leading to 


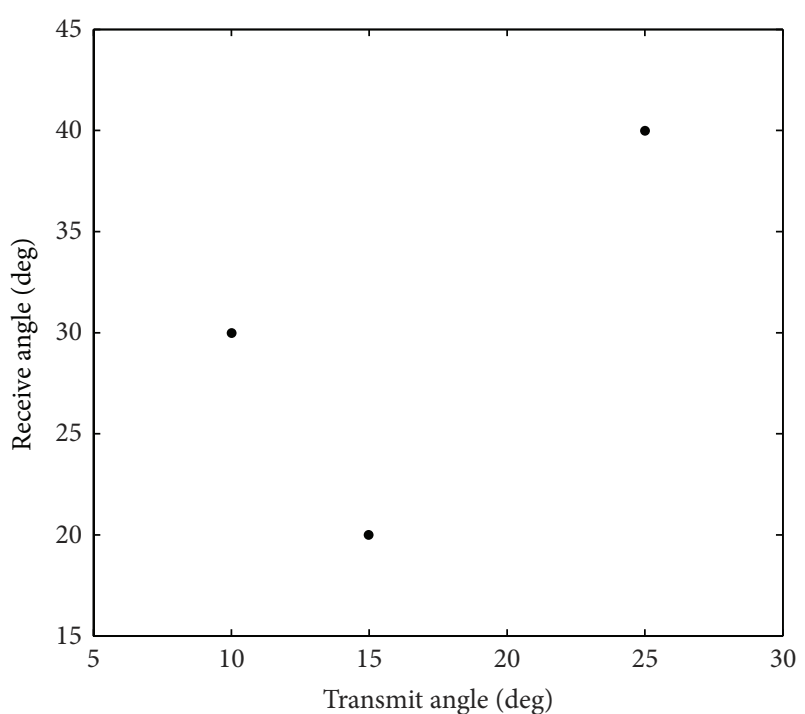

FIGURE 3: Paired results of proposed method for all three targets with $\mathrm{SNR}=0 \mathrm{~dB}$.

the high computational complexity. In order to reduce the computational cost of the algorithm, we propose the RDESPRIT-MUSIC algorithm.

In previous section, we construct the 2D-MUSIC null spectrum. And (11) is also denoted by

$$
\begin{aligned}
\left(\widehat{\mu}_{p}^{d}, \widehat{v}_{p}^{d}\right)=\underset{\mu_{p}^{(l)}, v_{p}^{(l)}}{\operatorname{argmin}}\left[\mathbf{a}\left(\mu_{p}\right)^{H}\left(\mathbf{a}\left(v_{p}\right) \otimes \mathbf{I}_{M_{t}}\right)^{H} \mathbf{E}_{n} \mathbf{E}_{n}^{H}\right. \\
\left.\quad \times\left(\mathbf{a}\left(v_{p}\right) \otimes \mathbf{I}_{M_{t}}\right) \mathbf{a}\left(\mu_{p}\right)\right] \\
=\underset{\mu_{p}^{(l)}, v_{p}^{(l)}}{\operatorname{argmin}}\left[\mathbf{a}\left(\mu_{p}\right)^{H} \mathbf{B}\left(v_{p}\right) \mathbf{a}\left(\mu_{p}\right)\right]
\end{aligned}
$$

where $\mathbf{B}\left(v_{p}\right)=\left(\mathbf{a}\left(v_{p}\right) \otimes \mathbf{I}_{M_{t}}\right)^{H} \mathbf{E}_{n} \mathbf{E}_{n}^{H}\left(\mathbf{a}\left(v_{p}\right) \otimes \mathbf{I}_{M_{t}}\right)$. Equation (12) is the problem of quadratic optimization. In order to eliminate the trivial solution $\mathbf{a}\left(\mu_{p}\right)=\mathbf{0}_{M_{t}}$, we add the constraint of $\mathbf{e}_{1}^{H} \mathbf{a}\left(\mu_{p}\right)=1$, where $\mathbf{e}_{1}=[1,0, \ldots, 0]^{T} \in R^{M_{t} \times 1}$. The optimization problem can be reconstructed as follows:

$$
\underset{\mu_{p}^{(l)}, v_{p}^{(l)}}{\operatorname{argmin}}\left[\mathbf{a}\left(\mu_{p}\right)^{H} \mathbf{B}\left(v_{p}\right) \mathbf{a}\left(\mu_{p}\right)\right], \quad \text { s.t. } \mathbf{e}_{1}^{H} \mathbf{a}\left(\mu_{p}\right)=1 .
$$

Then we construct the following costing function

$$
L\left(\mu_{p}, v_{p}\right)=\mathbf{a}\left(\mu_{p}\right)^{H} \mathbf{B}\left(v_{p}\right) \mathbf{a}\left(\mu_{p}\right)-\lambda\left(\mathbf{e}_{1}^{H} \mathbf{a}\left(\mu_{p}\right)-1\right),
$$

where $\lambda$ is constant. We have

$$
\frac{\partial}{\partial \mathbf{a}\left(\mu_{p}\right)} L\left(\mu_{p}, v_{p}\right)=2 \mathbf{B}\left(v_{p}\right) \mathbf{a}\left(\mu_{p}\right)-\lambda \mathbf{e}_{1}=0 .
$$

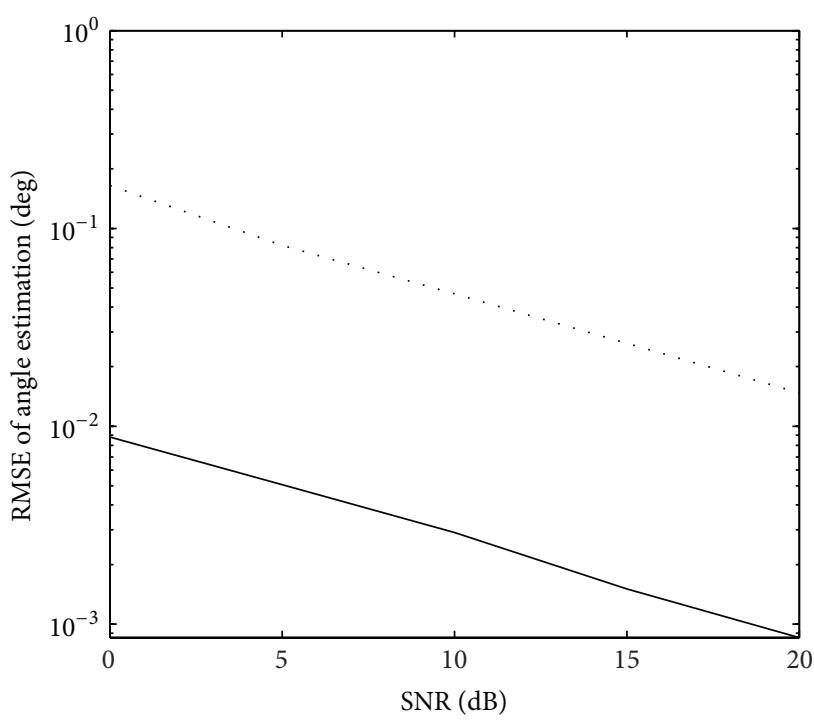

_ ESPRIT-MUSIC with sparse array

… ESPRIT with ULA

FIGURE 4: RMSE of angle estimation of the first target.

According to (15), $\mathbf{a}\left(\mu_{p}\right)=\mu \mathbf{B}^{-1}\left(\nu_{p}\right) \mathbf{e}_{1}$, where $\mu$ is constant. For $\mathbf{e}_{1}^{H} \mathbf{a}\left(\mu_{p}\right)=1, \mu=1 /\left(\mathbf{e}_{1}^{H} \mathbf{B}^{-1}\left(\nu_{p}\right) \mathbf{e}_{1}\right)$. So $\mathbf{a}\left(\mu_{p}\right)$ is obtained by

$$
\mathbf{a}\left(\mu_{p}\right)=\frac{\mathbf{B}^{-1}\left(v_{p}\right) \mathbf{e}_{1}}{\mathbf{e}_{1}^{H} \mathbf{B}^{-1}\left(v_{p}\right) \mathbf{e}_{1}} .
$$

Inserting $\mathbf{a}\left(\mu_{p}\right)$ of (16) into (12), then the receive angle cosine estimates are obtained by

$$
\widehat{v}_{p}^{d}=\underset{v_{p}^{(l)}}{\operatorname{argmin}} \frac{1}{\mathbf{e}_{1}^{H} \mathbf{B}^{-1}\left(v_{p}\right) \mathbf{e}_{1}}=\underset{v_{p}^{(l)}}{\operatorname{argmax}}\left[\mathbf{e}_{1}^{H} \mathbf{B}^{-1}\left(v_{p}\right) \mathbf{e}_{1}\right] .
$$

Searching $\nu_{p} \in v_{p}^{(l)}$, we can find the $P$ largest peaks $\left(\widehat{\nu}_{1}^{d}, \widehat{v}_{2}^{d}, \ldots\right.$, $\hat{v}_{P}^{d}$ ) which correspond to the receive angle. Then the receive angle $\varphi_{p}$ can be obtained by $\widehat{\varphi}_{p}=a \sin \left(\widehat{v}_{p}^{d}\right) *$ $180 / \pi$. Also, we can obtain the $P$ receive steering vectors $\left[\widehat{\mathbf{a}}\left(\nu_{1}\right), \widehat{\mathbf{a}}\left(\nu_{2}\right), \ldots, \widehat{\mathbf{a}}\left(\nu_{P}\right)\right]$. Inserting the receive steering vectors into $\mathbf{B}\left(v_{p}\right)=\left(\mathbf{a}\left(v_{p}\right) \otimes \mathbf{I}_{M}\right)^{H} \mathbf{E}_{n} \mathbf{E}_{n}^{H}\left(\mathbf{a}\left(v_{p}\right) \otimes \mathbf{I}_{M}\right)$, we obtain $\left[\widehat{\mathbf{B}}\left(v_{1}\right)\right.$, $\left.\widehat{\mathbf{B}}\left(\nu_{2}\right), \ldots, \widehat{\mathbf{B}}\left(\nu_{P}\right)\right]$. According to (12), we have

$$
\widehat{\mu}_{p}^{d}=\underset{\mu_{p}^{(l)}}{\operatorname{argmin}}\left[\mathbf{a}\left(\mu_{p}\right)^{H} \widehat{\mathbf{B}}\left(v_{p}\right) \mathbf{a}\left(\mu_{p}\right)\right] .
$$

Searching $\mu_{p} \in \mu_{p}^{(l)}$, the $P$ largest peaks $\left(\widehat{\mu}_{1}^{d}, \widehat{\mu}_{2}^{d}, \ldots, \widehat{\mu}_{P}^{d}\right)$ which correspond to the transmit angle can be obtained. Then the transmit angle $\theta_{p}$ can be expressed as $\widehat{\theta}_{p}=a \sin \left(\widehat{\mu}_{p}^{d}\right) * 180 / \pi$.

The main steps of Reduced-Dimension ESPRIT-MUSIC algorithm for angle estimation in the bistatic MIMO radar with sparse linear arrays as follows. 




FIGURE 5: RMSE of angle estimation of the first target with different L.

Step 1. Perform eigen-decomposition operation for the covariance matrix $\widehat{\mathbf{R}}$ to obtain $\mathbf{E}_{s}$ and $\mathbf{E}_{n}$.

Step 2. Calculate the ambiguous angle cosine estimates $\widehat{\mu}_{p}^{a}$ and $\hat{\nu}_{p}^{a}$ via (8).

Step 3. Find the set of cyclically related candidates $\mu_{p}^{(l)}$ and $\nu_{p}^{(l)}$ by (9).

Step 4. Searching $v_{p} \in v_{p}^{(l)}$ with respect to (17), the $P$ largest peaks $\left(\widehat{v}_{1}^{d}, \widehat{v}_{2}^{d}, \ldots, \widehat{v}_{P}^{d}\right)$ can be found. Then compute the receive angle $\varphi_{p}$.

Step 5. Searching $\mu_{p} \in \mu_{p}^{(l)}$ with respect to (18), the $P$ largest peaks $\left(\hat{\mu}_{1}^{d}, \widehat{\mu}_{2}^{d}, \ldots, \widehat{\mu}_{P}^{d}\right)$ can be obtained. Then calculate the transmit angle $\theta_{p}$.

4.2. Simulation Results. All simulations are implemented in the computer that consists of an Intel Core i3-2100 CPU at $3.10 \mathrm{GHz}$ and an internal memory with capacity of $2 \mathrm{~GB}$. Some simulations verify the angle estimation performance of RD-ESPRIT-MUSIC algorithm. To ensure fair comparison, all the experimental parameters are similar to Section 3.2. Assume that there exist $P=3$ uncorrelated stationary targets, which are located at angles $\left(\theta_{1}, \varphi_{1}\right)=\left(15^{\circ}, 20^{\circ}\right),\left(\theta_{2}, \varphi_{2}\right)=$ $\left(25^{\circ}, 40^{\circ}\right)$, and $\left(\theta_{3}, \varphi_{3}\right)=\left(10^{\circ}, 30^{\circ}\right)$, and the number of snapshots is $L=100$ for an $M_{t}=12, M_{s t}=3, N_{s t}=4$ and $M_{r}=$ $10, M_{s r}=2, N_{s r}=5$ bistatic MIMO radar. And the intersubarray spacing $d_{s}=30 *(\lambda / 2)$. From Figure 6 , we can see that the angle estimation performance of RD-ESPRIT-MUSIC algorithm is similar to the ESPRIT-MUSIC algorithm. And all of them are much better than ESPRIT with ULA. Figure 7

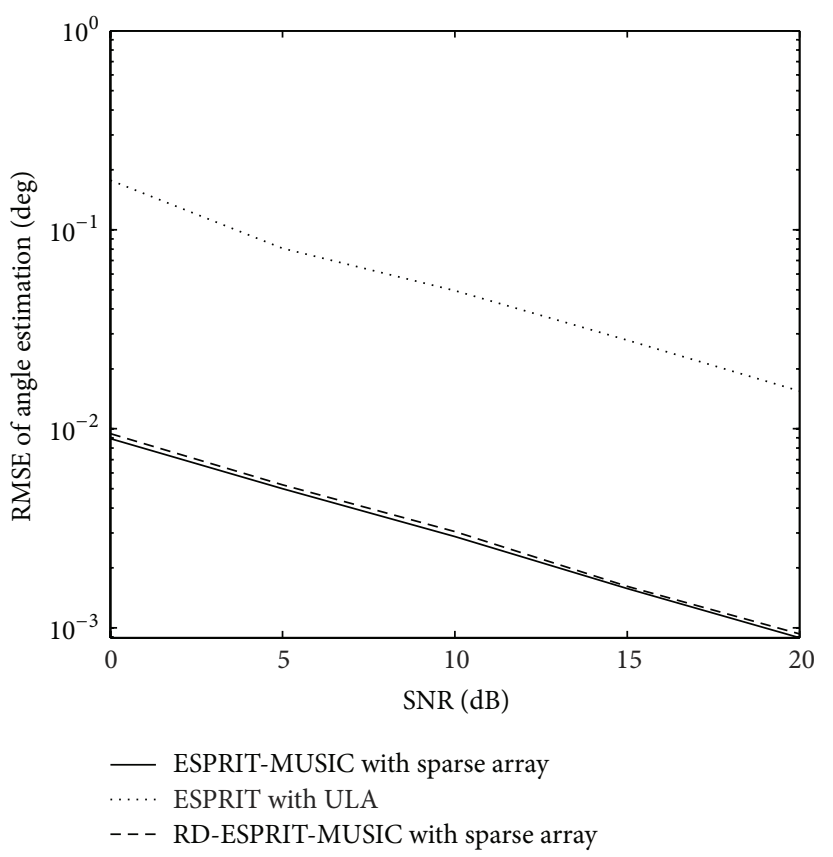

FIGURE 6: Angle estimation performance comparison.

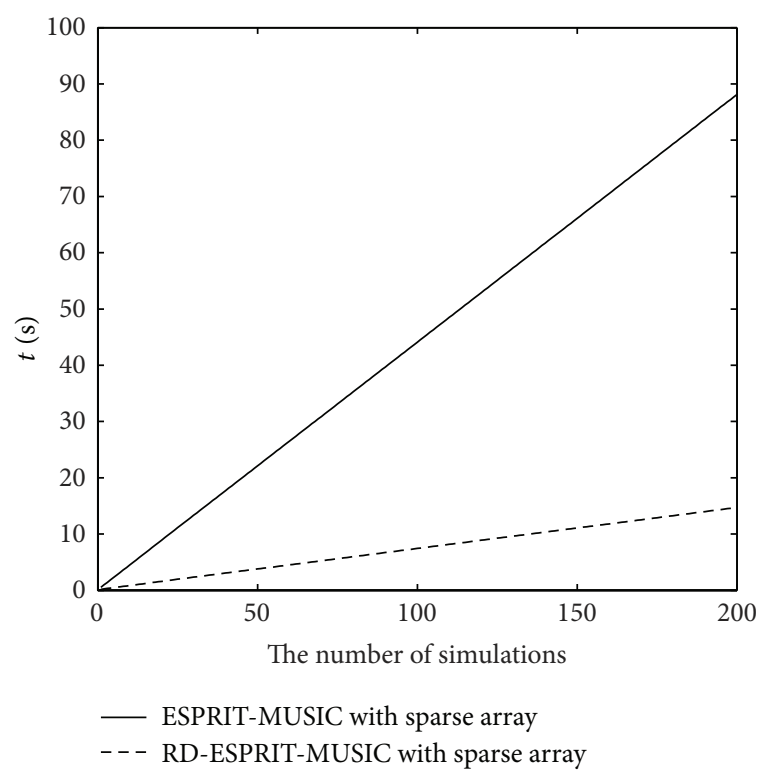

Figure 7: The runtime of algorithms comparison.

depicts the runtime comparison between ESPRIT-MUSIC algorithm and RD-ESPRIT-MUSIC algorithm. It is clearly shown that RD-ESPRIT-MUSIC algorithm pay much less time than ESPRIT-MUSIC algorithm. The simulation result demonstrates that the complexity of RD-ESPRIT-MUSIC algorithm reduces sharply.

\section{Conclusion}

In this paper, the ESPRIT-MUSIC method is proposed to estimate target angle in bistatic MIMO radar spaced sparse 
linear array. The proposed method can obtain the paired transmit angle and receive angle and avoid the manifold ambiguity. In order to reduce the computational complexity of the algorithm, we derive the RD-ESPRIT-MUSIC algorithm which only requires one-dimension search. All of them have much better performance than ESPRIT with ULA. Several simulation results are presented to verify the effectiveness of the two algorithms.

\section{Acknowledgments}

This work is supported by the National Natural Science Foundation of China (Grant no. 61172159) and the Fundamental Research Funds for the Central Universities (HEUCFT1101).

\section{References}

[1] E. Fishler, A. Haimovich, R. Blum, D. Chizhik, L. Cimini, and R. Valenzuela, "MIMO radar: an idea whose time has come," in Proceedings of the IEEE Radar Conference, pp. 71-78, Philadelphia, Pa, USA, April 2004.

[2] A. M. Haimovich, R. S. Blum, and L. J. Cimini, "MIMO radar with widely separated antennas," IEEE Signal Processing Magazine, vol. 25, no. 1, pp. 116-129, 2008.

[3] J. Li and P. Stoica, "MIMO radar with colocated antennas," IEEE Signal Processing Magazine, vol. 24, no. 5, pp. 106-114, 2007.

[4] E. Fishler, A. Haimovich, R. S. Blum, L. J. Cimini Jr., D. Chizhik, and R. A. Valenzuela, "Spatial diversity in radars-models and detection performance," IEEE Transactions on Signal Processing, vol. 54, no. 3, pp. 823-838, 2006.

[5] J. Li, P. Stoica, L. Xu, and W. Roberts, "On parameter identifiability of MIMO radar," IEEE Signal Processing Letters, vol. 14, no. 12, pp. 968-971, 2007.

[6] L. Xu, J. Li, and P. Stoica, "Target detection and parameter estimation for MIMO radar systems," IEEE Transactions on Aerospace and Electronic Systems, vol. 44, no. 3, pp. 927-939, 2008.

[7] C. Duofang, C. Baixiao, and Q. Guodong, "Angle estimation using ESPRIT in MIMO radar," Electronics Letters, vol. 44, no. 12, pp. 770-771, 2008.

[8] C. Jinli, G. Hong, and S. Weimin, "Angle estimation using ESPRIT without pairing in MIMO radar," Electronics Letters, vol. 44, no. 24, pp. 1422-1423, 2008.

[9] F. Gu, L. Chi, Q. Zhang, F. Zhu, and Y. Liang, "An imaging method for MIMO radar with sparse array based on Compressed Sensing," in Proceedings of the IEEE International Conference on Signal Processing, Communications and Computing (ICSPCC '11), pp. 1-4, Xi'an, China, September 2011.

[10] T. Strohmer and B. Friedlander, Analysis of Sparse MIMO Radar, Cornell University Library, 2012.

[11] D. Khan and K. L. Bell, "Analysis of DOA estimation performance of sparse linear arrays using the Ziv-Zakai bound," in Proceedings of the IEEE International Radar Conference (RADAR '10), pp. 746-751, Washington, DC, USA, May 2010.

[12] D. Khan and K. L. Bel, "Explicit Ziv-Zakai bound for DOA estimation with sparse linear arrays," in Proceedings of the 3rd IEEE International Workshop on Computational Advances in Multi-Sensor Adaptive Processing (CAMSAP '09), pp. 257-260, Dutch Antilles, The Netherlands, December 2009.
[13] D. W. Tufts, G. Hongya, and R. Kumaresan, "Resolving ambiguities in estimation spatial frequencies in sparse linear array," in Proceedings of the IEEE International Conference on Acoustics, Speech, and Signal Processing, pp. 345-348, Adelaide, South Australia, April 1994.

[14] W. Leng and A.-G. Wang, "Research of the ambiguity restraint in five-element cross-shaped array," in Proceedings of the International Conference on Microwave Technology and Computational Electromagnetics (ICMTCE '09), pp. 37-40, Beijing, China, November 2009.

[15] H.-L. Sun, H.-H. Tao, and R.-C. Hao, "Method of resolving ambiguity for sparse array via modified sparse even array based on MUSIC algorithm," in Proceedings of the 2nd Asia-Pacific Conference on Synthetic Aperture Radar (APSAR '09), pp. 246249, Huangshan, China, October 2009.

[16] F. Athley, "Optimization of element positions for direction finding with sparse arrays," in Bayesian Bounds for Parameter Estimation and NonlInear FilterIng/TrackIng, pp. 545-548, Wiley-IEEE Press, Hoboken-Piscataway, NJ, USA, 2007.

[17] H. Gazzah and K. Abed-Meraim, "Optimum ambiguity-free isotropic antenna arrays," in Proceedings of the IEEE International Conference on Acoustics, Speech, and Signal Processing (ICASSP '09), pp. 2157-2160, Taipei, Taiwan, April 2009.

[18] H. Gazzah, "Optimum antenna arrays for isotropic direction finding," IEEE Transactions on Aerospace and Electronic Systems, vol. 47, no. 2, pp. 1482-1489, 2011.

[19] V. I. Vasylyshyn and O. A. Garkusha, "Direction finding using sparse array composed of multiple identical subarrays," in Proceedings of the 5th International Conference on Antenna Theory and Techniques, pp. 273-276, Kyiv, Ukraine, May 2005.

[20] M. D. Zoltowski and K. T. Wong, "Closed-form eigenstructurebased direction finding using arbitrary but identical subarrays on a sparse uniform Cartesian array grid," IEEE Transactions on Signal Processing, vol. 48, no. 8, pp. 2205-2210, 2000.

[21] V. Vasylyshyn, "Direction of arrival estimation using ESPRIT with sparse arrays," in Proceedings of the 6th European Radar Conference (EuRAD '09), pp. 246-249, Rome, Italy, October 2009. 

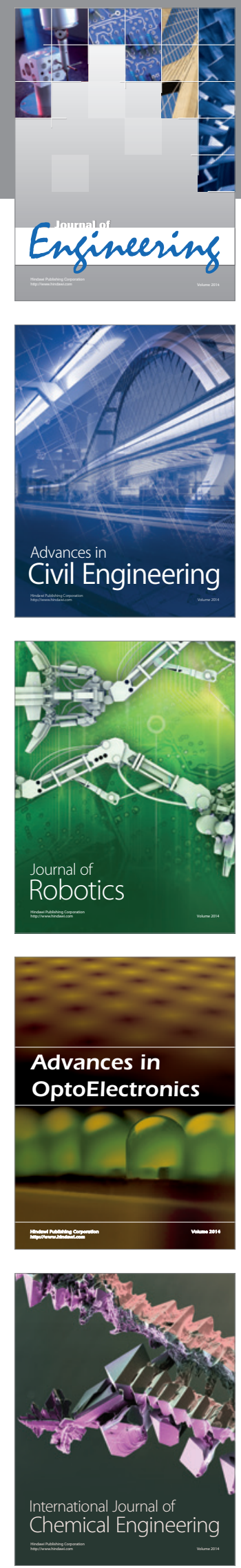



The Scientific World Journal


Submit your manuscripts at http://www.hindawi.com
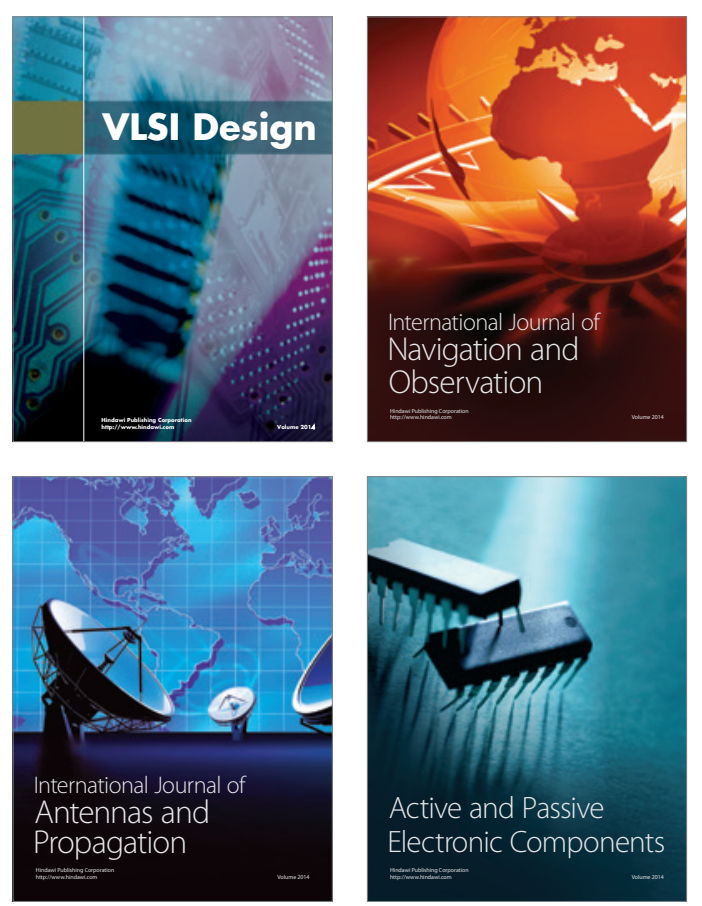
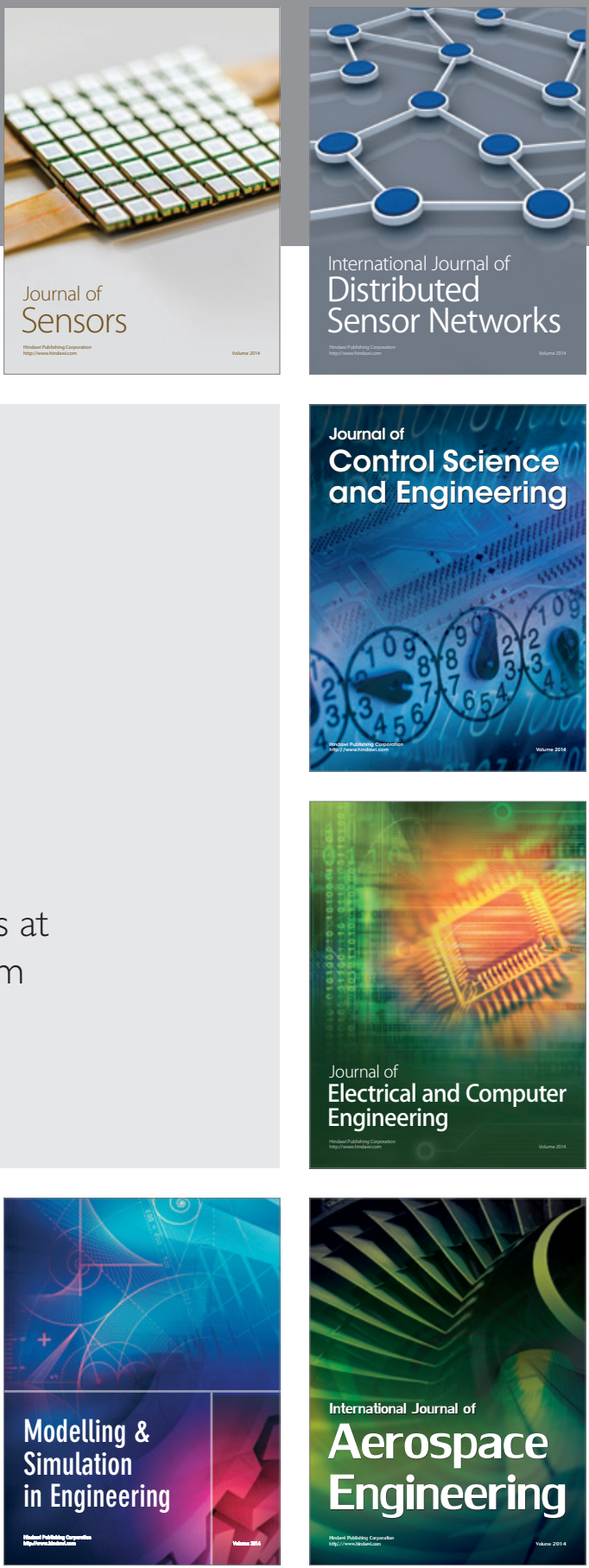

Journal of

Control Science

and Engineering
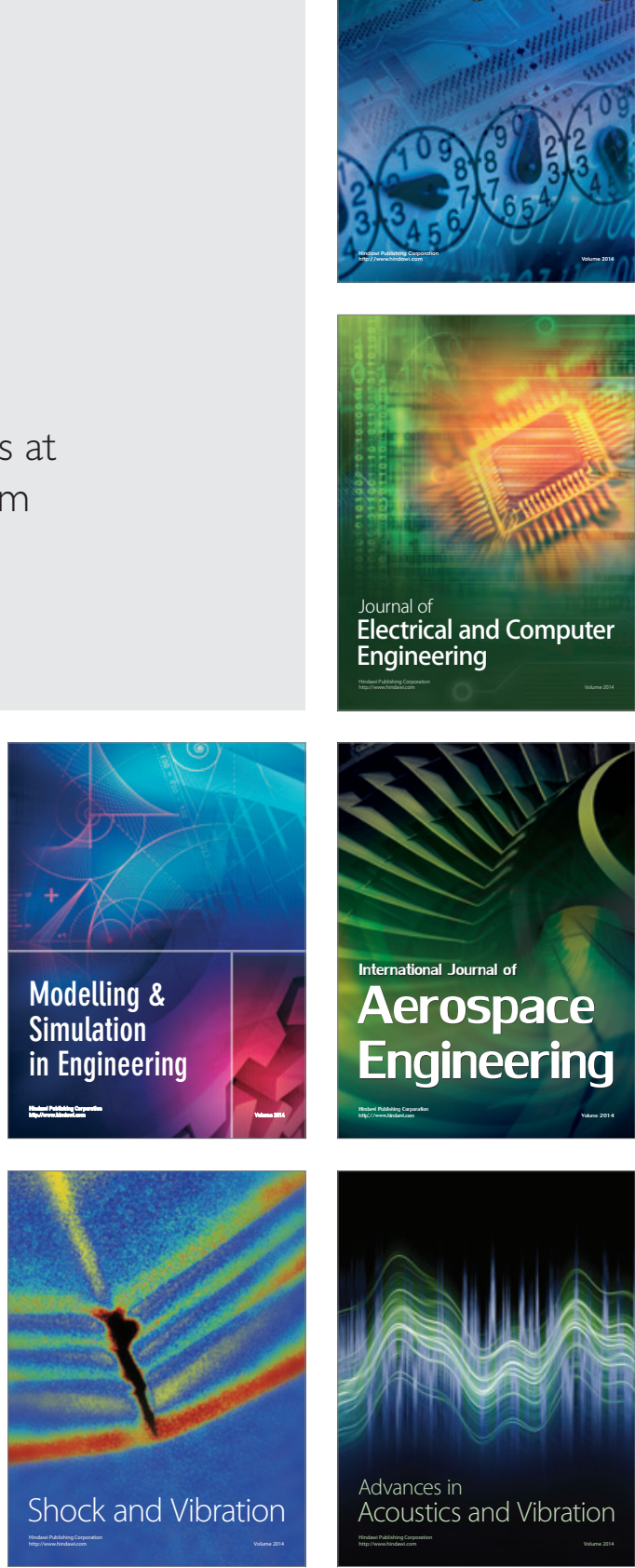